\title{
CARACTERIZAÇÃO DO MANUSEIO DO CATETER VENOSO CENTRAL TOTALMENTE IMPLANTADO EM UMA UNIDADE AMBULATORIAL QUIMIOTERÁPICA DE UM HOSPITAL UNIVERSITÁRIO
}

\section{CHARACTERIZATION OF THE HANDLING OF THE CENTRAL VENOUS CATHETER FULLY IMPLANTED IN A CHEMOTHERAPY OUTPATIENT UNIT OF A UNIVERSITY HOSPITAL}

\author{
Gabrielle Lemos Oliveira Rodrigues ${ }^{1} *$ Amanda Oliveira Monteles $^{1} *$ Andreia Farias Gomes $^{2}$
}

\section{RESUMO:}

Objetivo: Identificar a frequência e características no manejo e manutenção do cateter totalmente implantado em pacientes oncológicos. Metodologia: Trata-se de um estudo transversal, descritivo, de abordagem quantitativa. O estudo foi realizado na cidade de Fortaleza - CE, no ambulatório oncohematológico de administração de quimioterápicos e sintomáticos, pertencente a um hospital universitário. A coleta ocorreu entre os meses de outubro a novembro de 2020, realizada através das fichas de acompanhamento de pacientes que possuem o dispositivo da própria instituição. Os dados foram armazenados no programa Excel e efetuada a análise quantitativa com demonstração de frequência e percentual. O estudo seguiu os princípios éticos da pesquisa envolvendo seres humanos, resolução no 466/2012, do Conselho Nacional de Saúde, o projeto foi submetido ao Comitê de Ética em Pesquisa da instituição, obtendo parecer favorável. Resultados: A amostra foi constituída por 55 pacientes, destes $65,5 \%$ do sexo feminino, $74,5 \%$ possuíam diagnostico de doença hematológica, $45 \%$ encontrava-se em acompanhamento no setor de 1 a 5 anos. Foram puncionados o cateter 959 vezes, sendo 49,4\% puncionados para administração de quimioterápicos e 48,4\% para manutenção do dispositivo, os demais foram para antibioticoterapia e coleta de hemocultura. Ao realizar a aspiração do dispositivo, obteve retorno sanguíneo em 768 vezes, em 86 vezes o sangue refluiu com dificuldade e em 105 ocasiões não obteve retorno sanguíneo. Na grande maioria, 959 ocasiões, utilizou-se como forma de "locking" solução heparinizada Conclusão: Os resultados apresentados evidenciam a complexidade da assistência de enfermagem relacionada ao manuseio do cateter totalmente implantado.

Palavras chaves: Cuidados de enfermagem; Enfermagem oncológica; Cateter.

\begin{abstract}
Objective: To identify the frequency and characteristics in the management and maintenance of the fully implanted catheter in oncologic patients. Methodology: This is a transversal, descriptive, quantitative approach study. The study was carried out in the city of Fortaleza - CE, in the oncohematological outpatient clinic for the administration of chemotherapy and symptomatic drugs, belonging to a university hospital. The collection took place between the months of October and November 2020, through the monitoring forms of patients who have the device of the institution itself. The data were stored in the Excel program and the quantitative analysis was performed with demonstration of frequency and percentage. The study followed the ethical principles of research involving human beings, resolution No. 466/2012 of the National Health Council, the project was submitted to the Ethics in Research Committee of the institution, obtaining a favorable opinion. Results: The sample consisted of 55 patients, of these $65.5 \%$ were female, $74.5 \%$ had a diagnosis of hematological disease, $45 \%$ were under follow-up in the sector from 1 to 5 years. The catheter was punctured 959 times, $49.4 \%$ of which were for the administration of chemotherapy and $48.4 \%$ for maintenance of the device, the others were for antibiotic therapy and blood culture collection. When aspiring the device, it obtained blood return in 768 times, in 86 times the blood reflected with difficulty and in 105 occasions it did not obtain blood return. In the great majority, 959 times, heparinized solution was used as a form of "locking". Conclusion: The results presented show the complexity of nursing care related to the handling of the fully implanted cateter.

Keywords: Nursing Care, Oncology Nursing, Catheters

\footnotetext{
${ }^{1}$ Enfermeira. Residência em Assistência em Oncohematologia - Hospital Universitário Walter Cantídio / Universidade Federal do Ceará.

${ }^{2}$ Enfermeira. Mestre nos Cuidados Clínicos em Enfermagem e Saúde pela Universidade Estadual do Ceará. - Hospital Universitário Walter Cantídio / Universidade Federal do Ceará.
} 


\section{INTRODUÇÃO}

O câncer é considerado um problema de saúde em todo o mundo, pelo aumento dos novos casos a cada ano, exigindo dos sistemas de saúde investimentos nas ações de prevenção, tratamento, recuperação e de cuidados paliativos $^{(1)}$.

Apesar destes grandes investimentos, as bases de tratamento do câncer continuam alicerçadas nas principais modalidades: cirurgia, quimioterapia, imunoterapia, radioterapia. Dentre essas, a quimioterapia antineoplásica é a mais promissora modalidade no combate ao câncer ${ }^{(2)}$.

A quimioterapia consiste em uma terapêutica que utiliza agentes antineoplásicos capazes de controlar células malignas ou curar, sua administração pode ser via oral, subcutânea, intratecal, intramuscular, intravesical, intracavitária e a mais utilizada venosa, considerada também a mais segura, tanto em relação ao nível sérico da droga quanto em relação a sua absorção. Para administrá-los é imprescindível a obtenção de um acesso venoso seguro, pois sua infusão por via endovenosa periférica pode ser responsável por complicações locais como flebite, urticária, vasoespasmo, dor, edema, eritema, vesículas e hiperpigmentação ${ }^{(3)}$.
Dessa forma, é preciso optar por recursos que viabilizem um acesso venoso seguro, de longo prazo, o que pode ser obtido por meio do uso do Cateter Venoso Central Totalmente Implantado (CVC-TI), no qual possibilita a infusão prolongada de agentes antineoplásicos, com taxas de infecção menor, devido à redução de sua manipulação, reduz também o incômodo de múltiplas punções periféricas e não restringe as atividades dos pacientes, visto que é pequeno, fica alojado geralmente em locais que a vestimenta cobre o local ${ }^{(4)}$.

O enfermeiro tem importante papel no processo terapêutico de pacientes portadores do CVC-TI, visto que é o profissional habilitado para manipular o sistema de cateteres implantados, realizando punções, curativos e outros procedimentos. A durabilidade do cateter a longo prazo depende fundamentalmente destes cuidados ${ }^{(1)}$.

Os cuidados essenciais para a manutenção da permeabilidade desses cateteres incluem, utilizar técnica estéril sempre que acessar o dispositivo, puncionar a loja com agulha adequada, do tipo Huber, lavar com solução salina após a infusão de qualquer substância e ao término da infusão. Nos casos em que o cateter não é utilizado com frequência, o mesmo deve ser submetido 
à lavagem com solução salina e selo com solução de heparina ${ }^{(5)}$.

O estudo justifica-se pela necessidade de identificar potencialidades e fragilidades no manejo do cateter totalmente implantado, bem como, conhecer a rotina de manutenção do cateter em pacientes oncológicos, a fim de maximizar a segurança do paciente e a vida útil do Porth-a-cath.

O objetivo do estudo foi caracterizar o uso e manejo do cateter venoso central totalmente implantado em pacientes oncológicos de uma unidade ambulatorial quimioterápica de um hospital universitário. Diante do exposto, o presente estudo apresentou a seguinte questão norteadora: qual a caracterização do uso e manejo do cateter venosos central totalmente implantado em pacientes oncológicos de uma unidade ambulatorial quimioterápica de um hospital universitário?

\section{METODOLOGIA}

Trata-se de um estudo documental, transversal, de caráter descritivo, de abordagem quantitativa.

$\mathrm{O}$ estudo foi realizado na cidade de Fortaleza - CE, em um hospital universitário de referência municipal e estadual, no ambulatório de administração de quimioterápicos. A coleta ocorreu entre os meses de outubro a novembro de 2020.
A população foi constituída pelas fichas de acompanhamento de pacientes que possuem CVC-TI da própria instituição, essas fichas são preenchidas pelos enfermeiros e residentes de enfermagem do ambulatório de quimioterapia e ficam armazenadas no próprio setor. Foram incluídas fichas que continham os dados dos pacientes portadores desse tipo de cateter, bem como, a frequência e descrição das manutenções. Foram excluídas fichas incompletas no preenchimento dos dados e de pacientes menores de 18 anos. No setor existiam 67 fichas de acompanhamento do cateter central, mas foi utilizado apenas 55, devido inviabilidade das 12 , visto que faltavam informações básicas sobre o acompanhamento do paciente.

Foi utilizado a ficha de investigação da própria instituição, contendo algumas informações, tais como: diagnostico do paciente, data e local de inserção do cateter venoso totalmente implantado, data da primeira punção do cateter, motivo da punção, tipo de agulha utilizada, se o local apresenta sinais de infecção, características ao aspirar a solução que estava previamente no cateter, bem como características ao infundir soro fisiológico e medicações caso necessário, se ouve ou não realização de curativo, intervalo entre as manutenções ou administração de quimioterapia. 
Os dados foram transcritos para o formulário da pesquisa com as variáveis de interesse do estudo. Foram armazenados no programa Excel e efetuada a análise quantitativa com demonstração de frequência e percentual

Os dados foram analisados estatisticamente tomando por base as variáveis de interesse para o estudo e organizados em tabelas, sendo sua discussão realizada por meio da utilização de literatura pertinente à temática.

O estudo seguiu os princípios éticos da pesquisa envolvendo seres humanos, conforme resolução $n^{\circ} 466 / 2012$, outorgada pelo decreto $\mathrm{n}^{\text {o }}$ 93.933/87 do Conselho
Nacional de Saúde, que incorpora quatro preceitos básicos da bioética: autonomia, não maleficência, beneficência e justiça.

O projeto foi submetido ao Comitê de Ética em Pesquisa (CEP) da instituição de saúde, obtendo parecer favorável de 15 de setembro de 2020, com o número 4.279.193, CAAE: 36334420.6.0000.5045.

\section{RESULTADOS E DISCUSSÃO}

A partir da coleta e tabulação dos dados foi possível construir tabelas e realizar uma análise das informações, a fim de caracterizar o perfil do cateter venoso central totalmente implantado, o manejo e manutenção dos profissionais do serviço com esses dispositivos.

Tabela 1- caracterização dos participantes do estudo (N= 55). Fortaleza, CE, Brasil, 2020.

\begin{tabular}{l|l|l}
\hline VARIÁVEIS & N & \% \\
\hline Sexo & & \\
\hline Masculino & 19 & 34,5 \\
Feminino & 36 & 65,5 \\
\hline Diagnostico & & \\
\hline Doença hematológica & 41 & 74,5 \\
Tumores sólidos & 9 & 16,3 \\
Não especificado & 5 & 0,92 \\
\hline Tempo no serviço & & \\
\hline Até 1 més & 7 & 12,7 \\
$>1$ mès a $<1$ ano & 9 & 16,3 \\
$>1$ ano a $<5$ anos & 25 & 45,4 \\
$>5$ anos a <10 anos & 10 & 18,1
\end{tabular}




\begin{tabular}{l|l|l}
$>10$ anos & 4 & 0,7 \\
\hline TOTAL & $\mathbf{5 5}$ & $\mathbf{1 0 0 , 0 0}$ \\
\hline
\end{tabular}

Fonte: Elaborado pelas pesquisadoras, 2020.

A partir dos dados da primeira tabela, observa-se o sexo feminino como a maior parte da amostra, com a cifra de 36 mulheres $(65,5 \%)$ dos 55 pacientes e dentre os pacientes do sexo masculino foram 19 $(34,5 \%)$. Estudo na área corrobora com o resultado obtido, quando afirma que 58,4\% dos participantes do estudo de acompanhamento do port a cath eram do sexo feminino $^{(1)}$.

Em relação ao diagnóstico, $74,5 \%$ dos pacientes foram diagnosticados com doenças hematológicas, 16,3\% acompanhados por tumores sólidos e as fichas de cinco pacientes não continham o diagnóstico do cliente, ratifica-se a importância de registrar as informações completas nos documentos do cliente.

Em relação ao tempo de acompanhamento dos pacientes, é possível identificar que a maior parte dos pacientes se Tabela 2 - Diagnóstico clinico por quantidade de punções do cateter totalmente implantado. Fortaleza, CE, Brasil, 2020.

\begin{tabular}{l|l|l|l}
\hline Diagnostico & $\begin{array}{l}\text { Contagem de } \\
\text { DIAGNÓSTICO }\end{array}$ & $\begin{array}{l}\text { PUNÇÃO } \\
\text { PARA QT }\end{array}$ & $\begin{array}{l}\text { PUNÇÃO PARA } \\
\text { MANUTENÇÃO }\end{array}$ \\
\hline Ca De Reto & 1 & 5 & 0 \\
Coriocarcinoma & 1 & 6 & 2 \\
Leucemia Difusa De Grandes & 2 & 4 & 0
\end{tabular}

encontravam no serviço pelo período de 1 a 5 anos, com 25 pacientes $(45,4 \%)$. Em pesquisa realizada no assunto, a média de dias de acompanhamento a pacientes com o cateter totalmente implantado foi de 99 dias, variando de 1 a 605 dias $^{(1)}$. Observa-se que no presente estudo o tempo que os pacientes utilizam o dispositivo é prolongado em comparação a outros estudos.

No ambulatório, 4 pacientes até a coleta dos dados, estavam sendo acompanhados pelo uso do CVC-TI a mais de 10 anos, sendo a maior parte do acompanhamento realizando manutenções do cateter, 2 deles por 10 anos, 1 paciente sendo acompanhando por 15 anos e 1 deles sendo acompanhado por 17 anos. Outro estudo na área afirma em seu estudo que o CVC-TI pode ficar implantado e é utilizado por anos, por possuírem maior durabilidade e menor risco de infecção ${ }^{(4)}$. 


\begin{tabular}{|c|c|c|c|}
\hline Células B & & & \\
\hline Leucemia Linfoide Aguda & 3 & 28 & 23 \\
\hline $\begin{array}{l}\text { Leucemia Mieloide Aguda } \\
\text { Promielocitica }\end{array}$ & 2 & 16 & 5 \\
\hline Linfoma De Burkit & 1 & 0 & 20 \\
\hline Linfoma De Hodgkin & 25 & 306 & 321 \\
\hline $\begin{array}{l}\text { Linfoma De Hodgkin - } \\
\text { ESCLEROSE NODULAR }\end{array}$ & 1 & 12 & 23 \\
\hline $\begin{array}{l}\text { Linfoma Difuso De Grandes } \\
\text { Células B }\end{array}$ & 1 & 2 & 19 \\
\hline Linfoma Não Hodgkin & 5 & 19 & 15 \\
\hline Mieloma Multiplo & 1 & 18 & 0 \\
\hline Neo De Colon & 1 & 11 & 0 \\
\hline Neo De Mama & 4 & 24 & 16 \\
\hline Neo De Ovario & 1 & 9 & 1 \\
\hline Neo Gastrico & 1 & 9 & 0 \\
\hline Não Informado & 5 & 5 & 20 \\
\hline Total Geral & 55 & 474 & 465 \\
\hline
\end{tabular}

Fonte: Elaborado pelas pesquisadoras, 2020.

Dentro do diagnóstico clínico, as neoplasias hematológicas foram as mais prevalentes, justifica-se pelo o tratamento quimioterápico mais prolongado e com drogas mais vesicantes. Destes, 25 pacientes $(45,4 \%)$ representaram os linfomas de Hodgkin, seguido por outras doenças hematológicas, os linfomas não Hodgkin, leucemia mieloide aguda promielocitica, leucemia linfoide aguda e mieloma múltiplo. Dentre as doenças de tumores sólidos a mais prevalente foi câncer de mama, com 4 pacientes da amostra.
Um estudo realizado em um hospital de Minas Gerais, dos 36 participantes da pesquisa, 37,14\% apresentaram o diagnóstico de câncer de mama, seguido do câncer colorretal $(31,43 \%)$ e as neoplasias Esôfago, Leucemia, Linfoma de Hodgkin, Colo de útero, Ovário, Pâncreas, Pele não melanoma, Pulmão, Testículo e Vulva, com 1 paciente de cada diagnostico, contrastando com os resultados da presente pesquisa ${ }^{(6)}$.

Tabela 3 - Caracterização dos CVC- TI. Fortaleza, CE, Brasil, 2020. 


\begin{tabular}{|c|c|c|}
\hline VARIÁVEIS & $\mathbf{N}$ & $\%$ \\
\hline \multicolumn{3}{|l|}{ Punções } \\
\hline Punções para QT & 474 & 49,4 \\
\hline Punções para manutenção & 465 & 48,4 \\
\hline Punções para antibioticoterapia & 18 & 0,18 \\
\hline Punção para Hemocultura & 1 & 0,01 \\
\hline $\begin{array}{l}\text { Punção sem êxito para } \\
\text { terapêutica }\end{array}$ & 1 & 0,01 \\
\hline \multicolumn{3}{|l|}{ Inserção } \\
\hline Normal & 950 & 99,06 \\
\hline Com Hiperemia & 8 & 0,80 \\
\hline Com Secreção & 1 & 0,01 \\
\hline \multicolumn{3}{|l|}{ Aspiração } \\
\hline Reflui & 768 & 80.00 \\
\hline Reflui Com Dificuldade & 86 & 08,01 \\
\hline Não reflui & 105 & 10,9 \\
\hline \multicolumn{3}{|l|}{ Infusão } \\
\hline Infunde & 898 & 93,63 \\
\hline Infunde Com Dificuldade & 59 & 6.1 \\
\hline Não infunde & 2 & 0,20 \\
\hline \multicolumn{3}{|l|}{ Heparina } \\
\hline Com Heparina & 956 & 99,68 \\
\hline Sem Heparina & 4 & 00,41 \\
\hline TOTAL & 959 & 100,00 \\
\hline
\end{tabular}

Fonte: elaborado pelas pesquisadoras, 2020.

A punção do cateter venoso central totalmente implantado no ambulatório da pesquisa é utilizada para diferentes finalidades, seja para administração de quimioterápicos, coleta para hemocultura, antibioticoterapia além de manutenção do dispositivo, que na instituição é padronizado a cada três meses, caso o paciente não esteja utilizando o cateter com frequência. Um estudo na cidade do Rio de Janeiro foi identificado infecção do dispositivo em 14 pacientes, destes 5 apresentaram febre num 
total de 137 pacientes acompanhados na pesquisa $^{(1)}$.

Um estudo em um ambulatório hematológico pediátrico, também com fichas de acompanhamento do cateter venoso central totalmente implantado, com amostra de 78 pacientes, a coleta de sangue foi o motivo de manuseio do dispositivo que mais se destaca, com um total de 1.573 manipulações e o número de ativação e desativação do cateter alcançou um total de 1.119 manipulações ${ }^{(7)}$.

Boas práticas de uso aumentam a vida útil dos cateteres, minimizando a ocorrência de eventos adversos e complicações associadas, sejam elas mecânicas ou infecciosas. São inúmeras as contribuições da enfermagem para pacientes com esses dispositivos, tais como a punção segura, cuidados e troca do curativo, a manutenção do sistema fechado, são medidas imprescindíveis para a prevenção de infecção do cateter totalmente implantado, essas ações visam a uma assistência segura para o paciente oncológico, já tão fragilizado pela doença e tratamento $^{(8)}$.

Tal manutenção é realizada no ambulatório a cada 3 meses se não estiver em uso para demais finalidades, realizada antissepsia do local previamente com solução de clorexidina alcoólica, punção com agulha tipo Hubber, aspiração de $5 \mathrm{ml}$ de sangue do dispositivo, salinizado com $20 \mathrm{ml}$ de soro fisiológico, avaliando a permeabilidade do cateter, em seguida utiliza-se uma solução de heparina diluída, $1 \mathrm{ml}$ de heparina a $9 \mathrm{ml}$ de SF 0,9\%, infundindo $5 \mathrm{ml}$ desta solução e ao término retira-se a agulha, realiza curativo oclusivo e orienta-se ao paciente a não molhar a região e só retirar o curativo após 24 horas.

A manutenção do dispositivo deve ser realizada uma vez a cada 30 dias para troca da solução de heparina, que é utilizada para manter a permeabilidade do cateter $^{(7)}$, tempo esse bem menor do que praticado no setor do estudo.

Foram puncionados pelos profissionais do ambulatório o cateter venoso central totalmente implantado por 959 vezes, sendo 474 vezes $(49,4 \%)$ puncionados para administração de quimioterápicos, puncionado 465 vezes $(48,4 \%)$ para manutenção do dispositivo, $18(0,18 \%)$ vezes puncionado para administração de antibiótico, puncionado 1 vez para coleta de hemocultura e 1 vez puncionado o dispositivo sem êxito na terapêutica. A partir de uma única ativação do cateter venoso central totalmente implantado pode se fazer coleta de sangue, quimioterapia, manutenção, entre outras manipulações ${ }^{(7)}$.

$\mathrm{Na}$ grande maioria a pele da região de acesso do cateter venoso central totalmente implantado apresentou-se com o aspecto normal, foram 950 punções nessas condições $(99,06 \%)$, em 8 ocasiões a pele encontrava-se 
hiperemiada e em apenas 1 ocasião apresentou secreção na pele, o paciente referiu picos febris, no qual foi coletado hemocultura e indicado retirada do dispositivo.

A agulha utilizada em todas as punções do dispositivo foi a agulha tipo Hubber, própria para punção do cateter venoso central totalmente implantado, visto sua angulação e adentrar o dispositivo sem romper a camada de silicone do alojamento ${ }^{(8)}$.

Ao realizar a aspiração do dispositivo, utilizando uma seringa de $20 \mathrm{ml}$ contendo $10 \mathrm{ml}$ de SF 0,9\%, obteve retorno sanguíneo em 768 vezes (80\%), se ao aspirar não refluir conteúdo sanguíneo, tenta-se infundir aproximadamente $3 \mathrm{ml}$ de SF e logo em seguida aspira novamente, após essa técnica em 86 vezes $(08 \%)$ o sangue refluiu com dificuldade e em 105 ocasiões (10\%) não obteve retorno sanguíneo a aspiração.

Após checagem do posicionamento correto do dispositivo é infundido $20 \mathrm{ml}$ de SF $0,9 \%$ na técnica de push, em 898 vezes $(93,63 \%)$ o conteúdo foi infundido sem resistência, já em $59(6,1 \%)$ ocasiões os enfermeiros tiveram dificuldade ao infundir e por duas vezes não foi possível infundir o SF $0,9 \%$, no qual não foi tentado infundir demais medicações, foi retirado a agulha e solicitado que o paciente retornasse com um intervalo menor para a próxima manutenção do cateter.
Um estudo com 137 pacientes que possuem o cateter venoso totalmente implantado afirma que apenas 3 destes apresenta como complicação de uso e manuseio do dispositivo a ausência de fluxo e refluxo ao puncionar o cateter, orientando-se a retirada do dispositivo ${ }^{(1)}$. Oclusões podem ser causadas por: mau posicionamento do cateter, formação de dobras no trajeto, mau posicionamento da agulha no compartimento ou formação de depósitos de fibrina ou $\operatorname{trombos}^{(9)}$.

$\mathrm{Na}$ grande maioria, 959 ocasiões $(99,68 \%)$ utilizou-se como forma de "locking" solução heparinizada, contendo 1 $\mathrm{ml}$ de heparina diluída em $10 \mathrm{ml}$ de soro físiológico e infundindo $5 \mathrm{ml}$ desta solução. No serviço do presente estudo é rotina heparinizar o dispositivo sempre que puncionado. Deve-se retirar a solução de heparina administrada anteriormente, através da aspiração sanguínea, seguida da lavagem do cateter com $20 \mathrm{ml}$ de soro físiológico a $0,9 \%$ e infusão de nova solução de heparina no interior do cateter ${ }^{(4)}$.

Dentre as técnicas com o intuito de evitar obstruções encontra-se também a prática de realizar "flushs" de solução salina, de maneira vigorosa e pulsátil, promovendo turbulência no lúmen do cateter, para evitar a formação de detritos na parede do dispositivo, no qual deve ser realizado antes e depois da 
infusão de cada solução, medicamento ou transfusão de hemocomponentes ${ }^{(10)}$.

\section{CONCLUSÃO}

O cateter venoso central totalmente implantado tem sido utilizado nos serviços de oncologia, acompanhando a necessidade de tratamento prolongado, ofertando melhor qualidade de vida ao paciente. Os resultados apresentados evidenciam a complexidade que envolve a assistência de enfermagem relacionada ao manuseio do CVC-TI.

As evidências das contribuições da enfermagem apontadas neste artigo mostram que a punção segura, a manipulação do sistema conectado com o cateter venoso central totalmente implantado e manutenção são cuidados imprescindíveis para a prevenção de infecção do cateter totalmente implantado. Essas ações visam a uma assistência segura para o paciente oncológico, já tão fragilizado pela doença e tratamento.

Espera-se que esse estudo sinalize aos profissionais da enfermagem que as ações apontadas são simples e capazes de evitar danos ao paciente. Além disso, almeja-se que novas pesquisas na mesma temática possam ser desenvolvidas com abordagens metodológicas diferentes.

\section{REFERÊNCIAS}

1. Peixoto RMA, Souza SR, Silva JC, Mendes SEM, Soria DAC, Fontes LM. Complicações do cateter port a cath: subsídios para os cuidados de enfermagem. Revista Enfermagem Atual In Derme [Internet]. 2019 [acesso em 20 de dez de 2020]; 87. Disponível em: https://revistaenfermagematual.com.br/index. php/revista/article/view/169/71.

2. Brasil. Ministério da Saúde. Manual de Bases Técnicas da Oncologia - SIA/SUS Sistema de informações ambulatoriais. Brasília (DF); 2015.

3. Bertolazzi LG, Lanza MVC, Bitencourt EC, Canille RMS, Pereira LPS, Oliveira KA et al. Incidência e caracterização de reações adversas imediatas à infusão de quimioterápicos em hospital sentinela. Archives Health Sciences [Internet]. 2015 [acesso em 03 de set de 2020]; 22(3): [84-90]. Disponível em: http://www.cienciasdasaude.famerp.br/index. php/racs/article/view/107/114.

4. Zerati AE, Wolosker N, Luccia N, PuechLeão P. Cateteres venosos totalmente implantáveis: histórico, técnica de implante e complicações. J Vasc Bras [Internet]. 2017 [acesso em 20 de nov de 2020]; 16(2): [128139]. Disponível em: https://www.scielo.br/pdf/jvb/v16n2/16775449-jvb-1677-5449008216.pdf. 
5. VIEIRA NNP. Validação de manual de condutas para manuseio de cateter totalmente implantado [Dissertação]. Local: Universidade de Brasília; 2015.

6. Stefanutti R, Pereira NG, Ribeiro TM, Melo JLL, Petrini AL, Carvalho PCBF et al. Port-a-cath para Administração de quimioterapia sistêmica: conhecimento, adaptação/satisfação e complicações em pacientes oncológicos [Internet]. Brazilian Journal of health Review. 2020 [acesso em 25 de dez de 2020]; 3(4): [9926-9941]. Disponível em: https://www.brazilianjournals.com/index.php/ BJHR/article/view/14495.

7. Machado LBL, Moura DA, Cunha LBC, CUNHA KCS. Característica dos cateteres e de crianças portadoras de doença oncohematológica. Cogitare Enferm [Internet]. 2017 [acesso em 12 de nov de 2020]; 22(1): [01-11]. Disponível em: https://revistas.ufpr.br/cogitare/article/view/4 8448/pdf.

8. Oliveira TF, Rodrigues MCS. Enfermagem na prevenção de infecção em cateter totalmente implantado no paciente oncológico. Cogitare Enferm [Internet]. 2016 [acesso em 02 de dez de 2020]; 21(2): [0105]. Disponível em: https://docs.bvsalud.org/biblioref/2016/07/68 7/45523-182075-1-pb.pdf.
9. Pires NN, Vasques CI. Conhecimento de enfermeiros acerca do manuseio de cateter totalmente implantado. Texto Contexto Enferm [Internet]. 2014 [acesso em 01 de nov de 2020]; 23(2): [443-450]. Disponível em: https://www.scielo.br/pdf/tce/v23n2/pt_01040707-tce-23-02-00443.pdf.

10. Goossens GA. Flushing and locking of venous catheters: available evidence and evidence deficit. Nurs Res Pract [Internet]. 2015 [acesso em 16 de nov de 2020]. Disponível em: https://www.ncbi.nlm.nih.gov/pmc/articles/P MC4446496/.

Submissão: 2020-12-31

Aprovado: 2021-03-15 